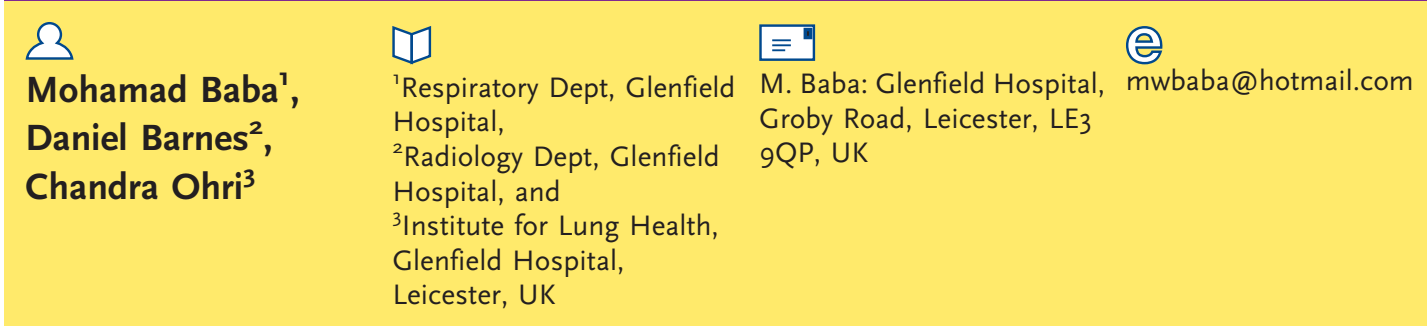

\title{
85-year-old patient with acute shortness of breath
}

\section{Case Report}

An 85-year-old male was admitted with a rapid increase in his shortness of breath, and blood-stained frothy sputum. His dyspnoea started just before going to sleep and, within a few hours, he became increasingly breathless. His past medical history included atrial fibrillation (on warfarin), aortic valve replacement (porcine), previous infective endocarditis, gout, hypothyroidism and hypertension. His medications on admission were warfarin alternating between 5 and $6 \mathrm{mg}$ once daily (q.d.), furosemide $120 \mathrm{mg}$ once monthly and $80 \mathrm{mg}$ q.d., levothyroxine $50 \mu \mathrm{g}$ q.d., allopurinol $400 \mathrm{mg}$ q.d., candesartan $16 \mathrm{mg}$ q.d., digoxin $125 \mu \mathrm{g}$ q.d.

On examination, the patient was in respiratory distress with a arterial oxygen saturation $\left(\mathrm{Sa}_{\mathrm{a}} \mathrm{O}_{2}\right)$ of $96 \%$ on $8 \mathrm{~L}$ of oxygen and a respiratory rate of 28 breaths. $\mathrm{min}^{-1}$ but was normotensive and afebrile. On auscultation he had diffuse bilateral crackles with raised jugular vein pressure. A chest radiograph (fig. 1) and chest computed tomography (CT) scan (fig. 2) were performed.

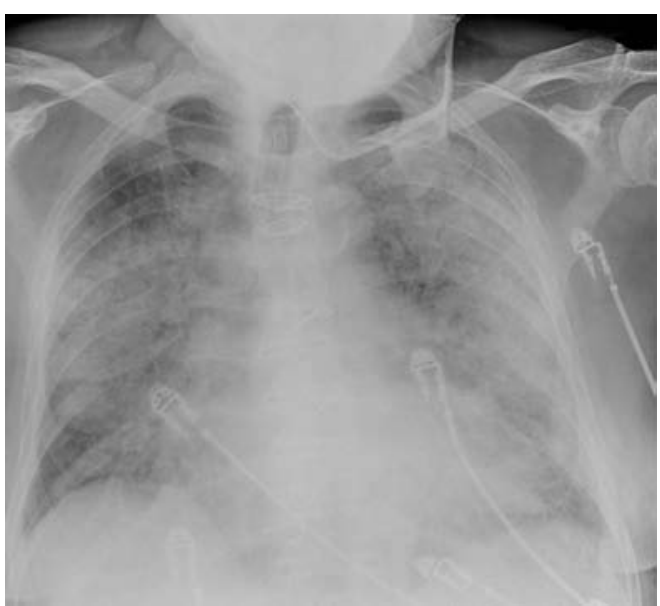

Figure 1

Chest radiograph.

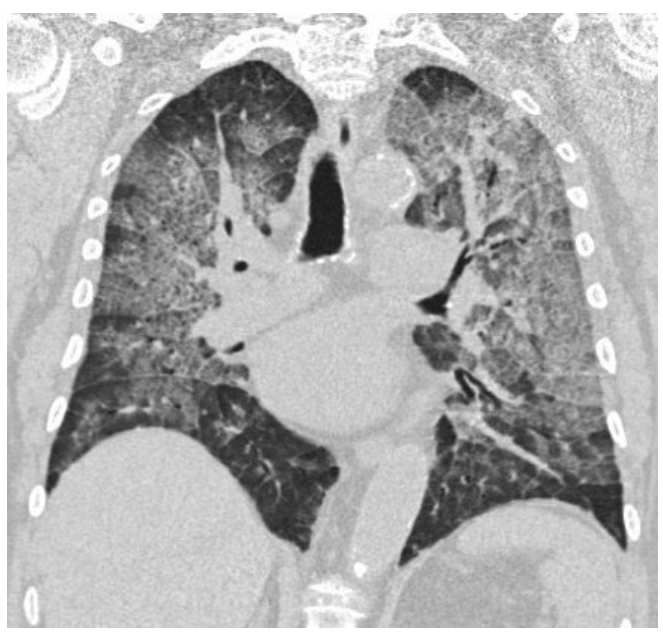

Figure 2

CT scan.
Statement of Interest None declared.

\section{Task 1}

How would you interpret this chest radiograph?

\section{Task 2}

What is your differential diagnosis according to the CT scan? 


\section{Answer 1}

There has been a previous sternotomy. There is widespread airspace shadowing in both lungs with some relative sparing of the apices and costophrenic angles. There are no significant pleural effusions.

\section{Answer 2}

There is widespread ground glass opacity with superimposed interlobular thickening giving a "crazy paving" appearance.
The presence of the interlobular thickening superimposed on the ground glass opacity results from haemosiderin and haemosiderin-laden macrophages accumulating in the interstitium. There are also associated areas of lobular sparing which are commonly seen in haemorrhage. From an imaging perspective, the "crazy paving" appearance is not specific to pulmonary haemorrhage and the differential is broad, depending on the time-course of the clinical picture. In this case, the acute history gives likely differentials of pulmonary haemorrhage, pulmonary oedema, atypical pneumonias and acute interstitial pneumonia/acute respiratory distress syndrome (ARDS).

His blood tests revealed an international normalised ratio (INR) $>10$ with a haemoglobin $(\mathrm{Hb})$ of $13.0 \mathrm{~g} \cdot \mathrm{dL}^{-1}$ and haematocrit of 0.391 . The patient was kept in the respiratory high-dependency unit for close monitoring. $\mathrm{His} \mathrm{Hb}$ decreased to $9 \mathrm{~g} \cdot \mathrm{dL}^{-1}$ in 4 days. An echocardiogram demonstrated normal left ventricle function and lung function tests revealed an increased transfer factor for $\mathrm{CO}$ (KCO; $107 \%$ predicted).

\section{Task 3}

What is your possible diagnosis according to clinical findings and imaging? 


\section{Answer 3}

Diffuse alveolar haemorrhage (DAH) is a life-threatening emergency leading to respiratory failure.

$\mathrm{DAH}$ is caused by damage to the alveolar capillary barrier or by disorders of coagulation $[1,2]$. Despite the increased risk of bleeding associated with anticoagulation, DAH is a rare complication, and there are only a few case reports describing the occurrence of $\mathrm{DAH}$ in patients on anticoagulation therapy. This condition is characterised by haemoptysis, falling haematocrit, respiratory failure and diffuse pulmonary infiltrates [3]. DAH is a rapid progressive life-threatening syndrome [4]. Early diagnosis is important in order to avoid any delay in appropriate management.

\section{Treatment}

With a high suspicion of DAH, the patient was started on intravenous vitamin K. However, given that heart failure and infection were differential diagnoses he also received intravenous furosemide, glyceryl trinitrate (GTN) infusion and fluid restriction. The patient was kept in the respiratory high-dependency unit for close monitoring. In the light of a possible superimposed infection or ARDS, the patient was started on intravenous antibiotics. Finally the patient started to improve slowly before being discharged from hospital with saturations of $97 \%$ on air after a 2 -week admission.

\section{Discussion}

$\mathrm{DAH}$ is a life-threatening syndrome that needs quick intervention. A bronchoalveolar lavage would have been the definitive diagnostic tool for DAH [5]; unfortunately it couldn't be done because our patient was in severe respiratory

\section{Task 4 \\ What are the aetiologies of $\mathrm{DAH}$ ?}

failure. The major differential diagnosis included $\mathrm{DAH}$, pulmonary oedema, infection and vasculitis. With hindsight and a number of normal investigations, we feel that the likely cause of this patient's presentation was DAH secondary to warfarin therapy. The diagnosis of DAH was considered as soon as the chest radiograph and initial INR blood test were performed. Despite initial intravenous vitamin $\mathrm{K}$ treatment the patient deteriorated and his $\mathrm{Hb}$ dropped whilst his inflammatory markers increased. Therefore, infection certainly could have been present, potentially as a direct consequence of $\mathrm{DAH}$. As the patient was too sick, a bronchoscopy on admission was not possible; however, a complete infection screen revealed no positive bacterial growth or virology. After 2 weeks of close monitoring and treatment, the patient became clinically stable and had lung function tests which revealed a high KCO which would support the diagnosis of DAH. Other conditions that might cause an elevated $\mathrm{KCO}$ include obesity, asthma, polycythaemia and left-to-right shunt [6], but our patient did not have any of evidence of these conditions.

This case emphasises the importance of making an early diagnosis, including differentials, in order to start appropriate treatment. Despite being a rare condition, DAH should be part of the differential diagnosis when a chest radiograph demonstrates diffuse bilateral shadowing. Warfarin-induced DAH is unusual, but given that more and more patients are now on long-term anticoagulation, presentations of this nature may become more frequent. 


\section{Answer 4}

The causes of DAH can be divided into two categories (table 1) [7-9]. Granulomatosis with polyangiitis (Wegener's) is the most common cause.
Table 1. Causes of DAH

DAH with vasculitis

- Idiopathic pulmonary haemosiderosis

- Systemic lupus

- Goodpasture's syndrome

- Diffuse alveolar damage

- Penicillamine

- Trimethyl anhydride

- Mitral stenosis

- Abnormal coagulation

- Lymphangioleiomatosis

- Tuberous sclerosis

DAH without vasculitis

- Granulomatosis with polyangiitis (Wegener's)

- Microscopic polyangiitis

- Connective tissue disease

- Antiphospholipid syndrome

- Mixed cryoglobulinaemia

- Behçet's syndrome

- Henoch-Schönlein Purpura

- Pauci-immune glomerulonephritis

- Glomerulonephritis with immune complex deposits

- Drug-induced

- Acute transplant rejection

\section{References}

1. Barnett VT, Bergmann F, Humphrey H, Chediak J. Diffuse alveolar hemorrhage secondary to superwarfarin ingestion. Chest 1992; 102: 1301-1302.

2. Waness A, Aldabbagh T, Harakati M. Diffuse alveolar haemorrhage secondary to warfarin therapy for atrial fibrillation: a case report and literature review. BMJ Case Rep 2009; 2009. pii bcro8.2008.0757.

3. Collard HR, Schwarz MI. Diffuse alveolar hemorrhage. Clin Chest Med 2004; 25: 583-592.

4. Thomas JK, Jayaprakash K, Misiriya KJR, et al. Catastrophic pulmonary alveolar hemorrhage complicating warfarin therapy. J Assoc Physician India 2008; 56: 34.

5. Rath GS, Schaff JT, Snider GL. Flexible fiberoptic bronchoscopy techniques and review of 100 bronchoscopies. Chest 1973; 63: 689-693.
6. Saydain G, Beck KC, Decker PA, et al. Membrane and capillary blood components of diffusion capacity of the lung for carbon monoxide in pulmonary sarcoidosis: relation to exercise gas exchange. Chest 2004; 125: 446-452.

7. Green RJ, Ruoss SJ, Kraft SA, et al. Pulmonarycapillaritis and alveolar hemorrhage. Update on diagnosis and management. Chest 1996; 110: 1305-1316.

8. Schwarz MI, Brown KK. Small vessels vasculitis of the lung. Thorax 2000; 55: 502-510.

9. Travis WD, Colby TV, Lombard C, et al. A clinicopathologic study of 34 cases of diffuse pulmonary hemorrhage with lung biopsy confirmation. Am J Surg Pathol 1990; 14: 1112-1125. 